\title{
Research on the Development Strategy of Big Health Industry in Jiangxi Province Based on SWOT
}

\author{
Nan Wang ${ }^{1}$, Lei $\mathrm{Wu}^{1, *}$, Jiayi Jiang ${ }^{2}$ and Yueping Zhou ${ }^{1}$ \\ ${ }^{1}$ School of Public Health, Nanchang University, Nanchang, China \\ ${ }^{2}$ Department of Computer Science, New York University \\ ${ }^{*}$ Corresponding author. Email: leiwu@ncu.edu.cn
}

\begin{abstract}
The big health industry is an emerging industry with great potential, which will lead the development of China's society and economy. This paper used SWOT model to analyze the strengths, weaknesses, opportunities, and challenges of the development of Jiangxi province's big health industry, and we put forward suggestions for the development of the big health industry to provide countermeasures for the development of Jiangxi province's big health industry.
\end{abstract}

Keywords: Big health industry, SWOT, Jiangxi Province.

\section{INTRODUCTION}

The big health industry is the "gold industry" and "hope industry" and "sunrise industry" leading the economic development and social progress in the $21 \mathrm{st}$ century and has become the focus of global attention. The big health industry is an integrated industry centered on the maintenance, repair, and promotion of health, which directly or indirectly provides healthy life solutions for the masses. It covers biomedicine, medical services, health tourism, health food, health care, pension and health management and other fields. Since the national "Thirteenth Five-Year Plan"[1] officially transformed the construction of a healthy China into a national strategy, Jiangxi province has also issued the 13th Five-Year Plan for the Development of the Major Health Industry [2], which proposes to take "medicine, medicine, tourism, food, nutrition and management" as the key points, to promote the integrated development of the major health industry and the construction of "healthy Jiangxi". This study uses the SWOT model (Strength, Weakness, Opportunity, and Threat) to analyze the strengths, weaknesses, opportunities, and threats of the development of Jiangxi province's big health industry, and provide suggestions for the development of Jiangxi province's big health industry.

\section{SWOT ANALYSIS OF BIG HEALTH INDUSTRY OF JIANGXI PROVINCE}

\subsection{Strength}

\subsubsection{Natural Resources}

Jiangxi province has picturesque scenery, and the quality of natural resources such as water, air, forest and agriculture is relatively good. In 2018, the province's forest coverage rate reached $63.1 \%$ [3], ranking second in China. In 2019 [4], Jiangxi had 75 provincial-level forest cities. Up to now, Jiangxi has 106 wetland parks at or above the provincial level, 190 nature reserves and 182 forest parks, with a wetland protection rate of $59.45 \%$. Jiangxi has good air quality, with 89.7 percent of days with good air quality in 2019, ranking 10th in China. In Jiangxi province, the ratio of excellent surface water examination section is $93.33 \%$, besides, there are abundant geothermal resources.

\subsubsection{Industrial Foundation}

By the end of the 12th Five-Year Plan period, the biomedical industry in Jiangxi province had realized the main business income of 125.8 billion yuan, and the total area of Chinese medicinal materials was about $700,000 \mathrm{mu}$. There were about 38,600 medical and health institutions of all kinds at all levels, with 4.33 beds in medical and health institutions for every 1,000 permanent residents [5]. The main business income of 
the green food industry exceeded 100 billion yuan. The total number of pollution-free agricultural products, green food, organic agricultural products, and geographical indications of agricultural products reached 2,902. Jiangxi has 6,430 home-based care centers, 1,781 institutions of various types and 193,000 beds for the aged.

\subsubsection{The Characteristic Brand}

Jiangxi province has its own characteristic pharmaceutical brands, especially traditional Chinese medicine brands. Now it has formed a number of wellknown brands, including 5 pharmaceutical groups: Jemincare, Renhe Pharmaceutical, Qingfeng Pharmaceutical, Huirin Pharmaceutical and Jiangzhong Pharmaceutical. Jiangzhong medicine valley has China's first Qhuang foreign political leaders experience center, which has received many foreign political leaders and effectively promoted the communication and exchange of traditional Chinese medicine culture. Heat-sensitive moxibustion technology is the original innovation achievement of Traditional Chinese medicine in Jiangxi Province. It won the second prize of National Science and Technology Progress Award. It has been applied and promoted in more than 500 hospitals in 27 provinces of China and opened overseas branches in Portugal. Zhangshu city of Yichun has a long history and is rich in Chinese medicinal materials, and it is renowned throughout the country. The brand awareness of green agricultural products in Poyang Lake is constantly improving.

\subsection{Weakness}

\subsubsection{Poor Industrial Agglomeration}

To establish a complete health industry system, it is necessary to integrate the six functions of prevention and health care, biopharmaceutical, health food, health care, leisure and tourism, and health management. However, there is no unified planning for the quality resources of health industry in Jiangxi province, and the integration and utilization of resources are poor, failing to form an industrial agglomeration effect. The development of health education, pharmaceutical manufacturing, medical services, elderly care, health enterprises and other industries is developed unbalanced and has not yet been well integrated.

\subsubsection{Single Investment and Financing Channels}

Smes are the largest and most dynamic group of enterprises, contributing more than $50 \%$ of the GDP and $70 \%$ of the scientific and technological innovation. However, due to various reasons, it is difficult for small and medium-sized enterprises in Jiangxi province to obtain bank credit support. They have narrow financing channels and high financing costs. At present, Jiangxi province lacks a diversified, socialized and sustainable health industry investment and financing system.

\subsubsection{Unitary Market}

China's health industry is still in its infancy. According to relevant data [6-8], the health industry accounts for $17.6 \%$ of GDP in the United States, $11.3 \%$ in Germany and $10 \%$ in Japan, which are the pillar industries of their countries. According to preliminary statistics, China's health industry accounts for about $6 \%$ of GDP, and Jiangxi province only accounts for about $5.8 \%$. Medical care in Jiangxi accounted for 8.8 percent of residents' per capita consumption in 2019. By the end of 2019 , there were $5.123,000$ people $(11.0 \%)$ over 65 years old in Jiangxi province, and the consumer groups of the health industry were mainly middle-aged and elderly people and chronic patients [5]. The consumers of health industry in Jiangxi province are relatively unitary.

\subsubsection{Shortage of Professional Talents}

The big health industry is a new sunrise industry, but Jiangxi province is short of related talents in health industry now. According to statistics [5], in 2018, the health technicians per 1,000 people in Jiangxi province were 5.32, practicing (assistant) physicians were 1.88, and registered nurses were 2.39, still far behind those in provinces with better health resources.

\subsection{Opportunity}

\subsubsection{Changes in Market Demand}

With the rapid improvement of living standards, people's health awareness has been constantly enhanced, and their health needs will be transformed from a single medical service demand to disease prevention, health promotion, health rehabilitation and other diversified needs. The continuous improvement of the social security system will increase people's demand for health.

\subsubsection{Technology Development}

A series of high-tech breakthroughs and applications have greatly promoted the rapid development of the big health industry. With the widespread use of "Internet Plus" and a new generation of information technology, Internet companies and smartphone makers are gradually infiltrating the healthcare sector. Now there are new modes of health services such as remote diagnosis, intelligent medicine, and individualized treatment. 


\subsubsection{Policy Support}

The "Healthy China 2030" Program provides a rare historical opportunity for the development of the big health industry. Jiangxi province established the national ecological civilization pilot zone and listed the great health industry as one of the key industries to realize leapfrog development. A series of policies and measures have been formulated to accelerate the development of industries, providing a strong guarantee for the development of the great health industry.

\subsection{Threat}

\subsubsection{Competition from Surrounding Provinces}

Jiangxi is the only province adjacent to the Pearl River Delta, Yangtze River Delta and The West of the Economic zone. Due to the fact that Jiangxi lags behind surrounding provinces in economic development to some extent, such as Zhejiang and Guangdong, the great health industry in Jiangxi started late, with incomplete industrial structure and low overall level. To a certain extent, Jiangxi lacks the attraction of retaining professional talents.

\subsubsection{Incomplete Health Industry Laws and Policies}

The great health industry is both a traditional and a new industry in Jiangxi Province, and its effective operation needs special policy environment and laws and regulations to guarantee. At present, laws and regulations related to the development of the health industry have not been improved, and the implementation of supervision and financial input still needs to be improved.

\section{CONCLUSION}

Through SWOT analysis, there are many deficiencies in Jiangxi Province's big health industry, especially the unbalanced development and imperfect related policies. The next stage will focus on achieving all-round development.To develop the health industry in Jiangxi province, it is necessary to make full use of the policy advantages of national support to the health industry. Considering the development needs and current situation of Jiangxi Province, Jiangxi province should make full use of existing resources, industries and brand advantages to promote the in-depth integration of information technology such as Internet and block chain with health industry. Jiangxi province should cultivate innovative talents, encourage scientific and technological innovation, and form industrial clusters. The general health industry should focus on medicine, medical care, tourism, food, elderly care and health management to build a comprehensive industrial chain. At the same time, relevant policies will be introduced to protect the development of the health industry.

\section{AUTHORS' CONTRIBUTIONS}

L.W. contributed to the conception of the study and the funding acquisition of the project. N.W. and J.J. contributed significantly to manuscript preparation (including data collection.); N.W. wrote the manuscript and contributed to the creation or presentation of this paper. Y.Z. helped perform the analysis with constructive discussions.

\section{ACKNOWLEDGMENTS}

This work was supported by the Project Supported by Jiangxi Provincial Natural Science Foundation of China [grant number 20192BAA208005]

\section{REFERENCES}

[1] Chinese Government website. The CPC Central Committee and the State Council issued the "Healthy China 2030" Plan Outline. 2016-10-25.

[2] The People's Government of Jiangxi Province. The People's Government of Jiangxi Province issued the Notice on the Development Plan of the Big Health Industry in the 13th Five-Year Plan of Jiangxi Province. 2017-3-17.

[3] Jiangxi Provincial Greening Committee. Jiangxi Province's Greening Status Bulletin for 2018 2019-3-12.

[4] Jiangxi Provincial Greening Committee. Jiangxi Province's Greening Status Bulletin for 2019. 2020-3-12.

[5] National Bureau of Statistics. Statistical Bulletin of the National Economic and Social Development of the People's Republic of China 2019. 2020-2-28

[6] Organisation for Ecomomic Cooperation and Development. Health Expenditure and Financing. 2019-04-09.

[7] Castro M C,Wilson M E,Bloom D E.Disease and economic burdens of dengue, The Lancet Infectious Diseases,2017,17(3):e70-e78. https://doi.org/10.1016/S1473-3099 (16)30545-X

[8] The World Bank. Current health expenditure (\% of GDP). 2019-04-09 